\title{
Feature Extraction of Plant Electrical Signals Based on Wavelet Transform
}

\author{
Zhang Xiaohui, Zang Haihe, Su baoping and Zhang zhixia \\ ${ }^{1}$ College of Electrical Engineering, Henan University of Technology, Zhengzhou \\ 450001, China \\ ${ }^{2}$ School of Information Engineer, Henan Institute of Science and Technology, \\ Xinxiang 453003, China \\ Correspondence author: xiaohui.xh@163.com
}

\begin{abstract}
A method of feature extraction in non-stationary signals is proposed to improve the correct classification rates of plant electrical signals. The samples are composed of plant electrical signals datum which includes the period of four circumstances. For wavelets have the trait of arbitrary distinction and decomposition, eigenvector which reflect different state of plant electrical signals are extracted from different frequency segments with the technology of wavelet decomposition. Then we take them input neural network as samples to establish the model of BP neural network. Experimental results demonstrate that the classification accuracy of the proposed feature extraction method for experiment plant electrical signals is high.
\end{abstract}

Keywords: plant electrical signals, wavelet transform, feature extraction, BP neural network

\section{Introduction}

Plant electrical signal, the transmitted information related to the processes of plant physiology is an important physiological signal. It is the plant in response to environmental change stimulation. Scientists from verify the presence of radio signals of the plant to ascertain the characteristics and electrical transmission mechanisms of the plant, and development to the current model for establishing electrical plants and related physiological effects study [1-5]. With the development of weak signal detection processing technology and computer information collection and data processing system realization, the extraction and analysis of weak plant electrical signal will be more scientific; feasibility and quantitative analysis of the wave signals of the plant is also possible. There are commonly used methods $[6,7]$ : plants wave signals in time domain, frequency domain analysis, wavelet analysis and time series analysis, electrical plants AR model and neural network prediction, and so on.

Wavelet transform signal projected onto a set of mutually orthogonal wavelet function sheets into space, which can be decomposed into two parts: low frequency part and high frequency part. In the next layer decomposition, the low frequency part was conducted re-decomposition, while the high frequency part was not conducted re- decomposition, which is very suitable for the weak low-frequency electrical signal feature extraction of the plant. Based on wavelet transform, this paper presents a method, which is suitable for non-stationary signal feature extraction, and wavelet coefficients energy eigenvalues as BP neural network classifier for automatic identification, achieving good recognition effect. 


\section{Basic Principles}

\subsection{Wavelet Decomposition Principle}

For the finite length signal $f(t)$ with noise, a suitable wavelet function is selected as the wavelet transform:

$$
\left(W_{\psi} f\right)(a, b)=\left\langle f, \psi_{a, b}\right\rangle=|a|^{-\frac{1}{2}} \int_{-\infty}^{+\infty} f(t) \psi^{*}\left(\frac{t-b}{a}\right) \mathrm{d} t
$$

where $a, b \in \mathrm{R}, a \neq 0, a$ is scaling factor, $b$ is a displacement factor, and $\psi^{*}$ is the conjugate operation of $\psi$.

The wavelet coefficients are processed by soft threshold in the wavelet transforming domain. When selecting the threshold, the standard deviation of original signal's noise must be estimated. In this study, we used equation (2) to estimate noise deviation.

$$
\sigma=\frac{\operatorname{median}\left(\left|d_{j}(k)\right|\right)}{0.6745} \quad\left(k=0,1, \square, 2^{j}-1\right)
$$

where $j$ is the wavelet decomposition scale, and median is the Matlab command. The Donoho threshold estimation formula is then used to calculate the threshold.

$$
\operatorname{thr}=\sigma \sqrt{2 \log (n)}
$$

where $n$ is the length of signal.

Then, wavelet coefficients are determined by using the wavelet de-noising soft threshold rules. When the absolute value of wavelet coefficients is less than the threshold, the wavelet coefficient is zero. Otherwise, the wavelet coefficient is the value of subtracting the threshold from the absolute value of wavelet coefficients which is greater than the threshold. This is summarized in equation (4):

where $d_{j, t h r}$ is the value of the wavelet coefficient processed by the threshold. Finally, the signal $f(t)$ is obtained with theinverse wavelet transform as follows:

$$
d_{j, t h r}=\left\{\begin{array}{c}
0,\left|d_{j}\right|<t h r \\
\operatorname{sgn}\left(d_{j}\right)\left(\left|d_{j}\right|-t h r\right),\left|d_{j}\right| \geq t h r
\end{array}\right.
$$

where $C_{\psi}=\int_{-\infty}^{+\infty}|\omega|^{-1}|\hat{\psi}(\omega)|^{2} \mathrm{~d} \omega<\infty, \hat{\psi}(\omega)$ is the Fourier transform of $\psi(t)$, and $f(t)$ is the result with inverse wavelet transform and reconstruction.

It is an effective tool for signal processing because wavelet transform can localize in both time and frequency field. In practical, we often use Mall at fast algorithm. Here will be described with three-layer decomposition, and wavelet decomposition tree shown in Figure 1. Where, $\mathrm{S}$ is the signal to be decomposed, representing the decomposed approaching signal of $A_{n}(n=1,2,3, \mathrm{~L}) . D_{n}(n=1,2,3, \mathrm{~L})$ represents the details of the decomposed signal[8]. 


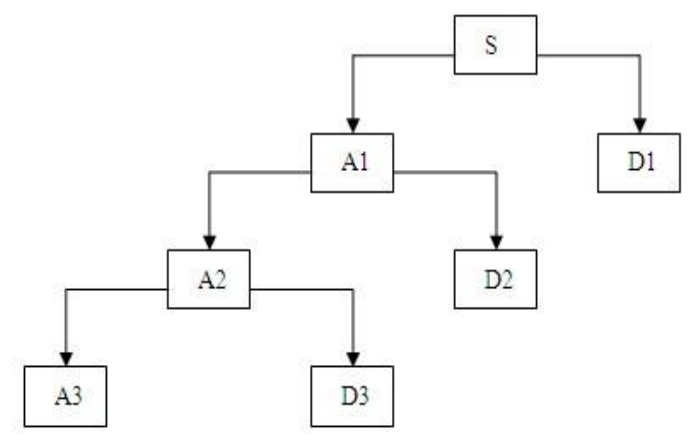

Figure 1. Three-level Structure of the Wavelet Decomposition Tree

As can be seen in Figure 1, the low frequency part was further decomposed, but the high frequency part will not be decomposed. Decomposition has relation $\mathrm{S}=\mathrm{A} 3+\mathrm{D} 3+\mathrm{D} 2+\mathrm{D} 1$. In addition, there is just explaining three-layer decomposition. If we want to further decompose, the low-frequency part of the A3 can be decomposed into low frequency A4 and high frequency D4. The following further decomposition, and so on.

\subsection{Electric Signal of the Plant}

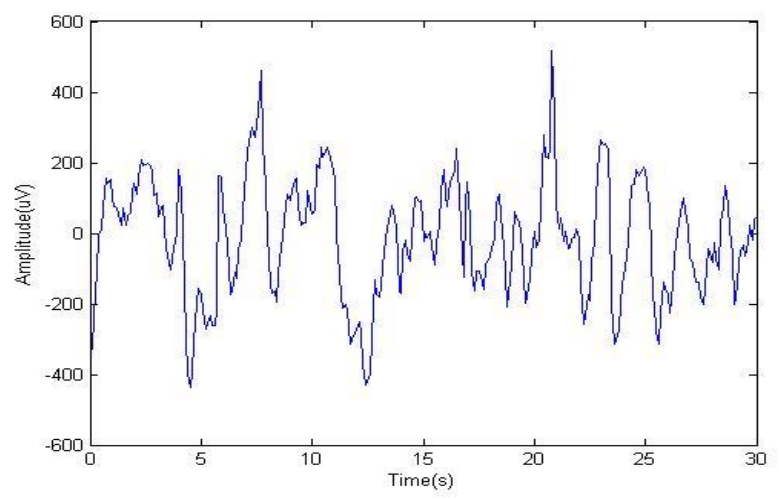

Figure 2. Electric Signal of the Plant

The electric signal of the plant in Figure 2 is weak low-frequency. Studies [9, 10] showed that the amplitude of electric signal of the plant is only a few $\mathrm{pV}$ to a few $\mathrm{mV}$ range. But in the normal physiological activity of the plant is only microvolt $(\mu \mathrm{V})$.Animals and people can touch the pulse beat to reach millivolts $(\mathrm{mV})$ level has already a scientific conclusion. Humans are unable to touch the pulse beat of the plant, maybe some sensitive plant such as mimosa and Venus flytrap, etc., may reach this magnitude in an instant after the stimulation.

Experimental data is the electric signal of aloe Vera collected under the darkness, broken branches, smoke, and burning conditions or stimuli condition. In this study, We used the biological function experiment system (designed by the VLSI laboratory of Henan University of Technology) to measure electrical signals. The input impedance is $400 \mathrm{M} \Omega$, and every input channel is photo electrically isolated, which decreases outside interference. The equivalent input noise voltage of the instrument was less than $1 \mu \mathrm{V}$. The measuring system is shown in Figure 3 . The constant temperature control system, shielding box, and measuring instrument were connected to each other. The sampling frequency was $5 \mathrm{kHz}$, the frequency of the low-pass filter was $500 \mathrm{~Hz}$, and that of the high-pass filter was $0.053 \mathrm{~Hz}$. The unit of electrical potential 
energy was $\mu \mathrm{V}$,the unit of time was second. During sampling, the temperature of the environment was $20^{\circ} \mathrm{C}$ and the humidity was $40-50 \%$.

The electrode was a high-sensitivity silver AMBU ECG electrode. Before the experiment, a test of the electrodes showed that they detected $0-50 \mathrm{~Hz}$ signals with fluctuations of less than $0.5 \%$, demonstrating high electrode stability. To measure the plant electrical signal, the A electrode was placed on the front surface of the leaf and the $\mathrm{B}$ electrode on the back (leaf length, $25 \mathrm{~cm}$; leaf width, $2 \mathrm{~cm}$ ). The distance between two electrodes was $15 \mathrm{~cm}$. The $\mathrm{C}$ electrode was connected to the copper sheet, which wasin the soil. The measuring electrode was connected to the signal measurement system, and the electrical signal was monitored and recorded by a computer. The environment temperature was regulated by the constant temperature controller[11].

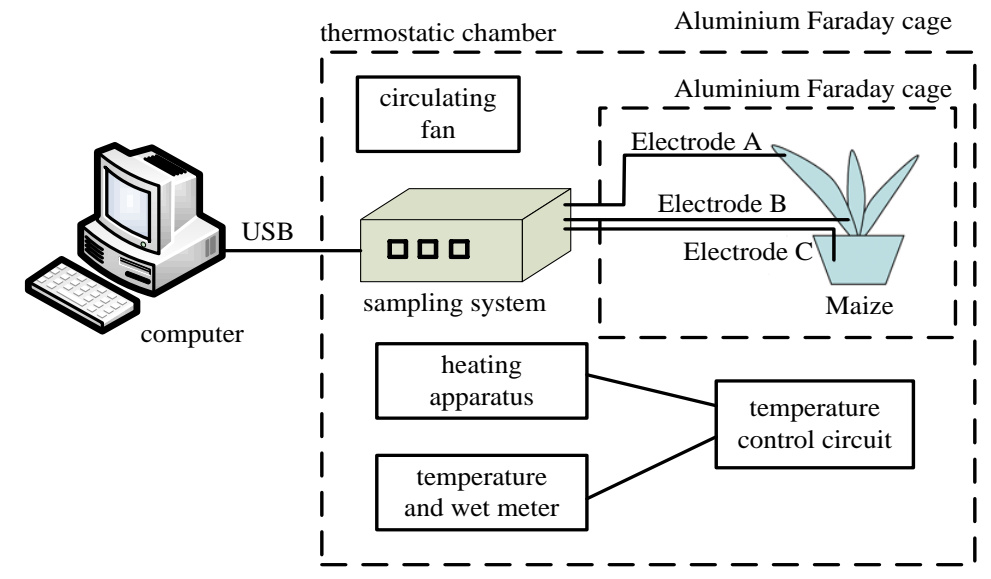

Figure 3. Schematic Diagram of Experimental Device

In the experiment, its basic operating principle is: firstly, through the lead electrodes lead the electric signal of the plant, which are obtained by the test electrodes, and amplify, filter, and other process the electrical signal. Secondly, the processed signal is digitized by analog to digital conversion and transfer to the computer via USB cable [11, 12].The sampling frequency of the original data is $F_{s}=5000 \mathrm{~Hz}$. After wavelet decomposition, the n-lever detail coefficients corresponding to frequency range is $\left(F_{s} / 2^{n+1}, F_{s} / 2^{n}\right)$; the $\mathrm{n}$-lever approximation coefficients corresponding to frequency range is $\left(0, F_{s} / 2^{n+1}\right)$. Thus, we can calculate the details of the wavelet decomposition of the signal and the approximation signals' the frequency range, which are shown in Table 1.

Table 1. Frequency Range after Wavelet Decomposition

\begin{tabular}{|c|c|c|c|c|c|c|}
\hline Signals & D5 & d4 & d3 & d2 & d1 & a5 \\
\hline Frequency range & $156.75 \sim$ & $312.5 \sim$ & $625 \sim$ & $1250 \sim$ & $2500 \sim$ & $0 \sim$ \\
$(H Z)$ & 312.5 & 625 & 1250 & 2500 & 5000 & 156.75 \\
\hline
\end{tabular}




\subsection{BP Neural Network Establishment and Training}

BP neural network has been successful in many applications of recognition and classification problems. BP network is a former propagation multilayered network, in addition to the input layer and the output layer, there are one or more hidden layers. The network training method is called error back propagation method (Back Propagation, BP), which uses the minimum error between the actual network output and the desired output(target vector) to correct the multilayer network connection weights layer by layer from back to front. This successive constantly modify the weight vector method called successive correction method [13].

In practical applications, the number of nodes in the input layer should be determined according to the dimension of the input data; the number of nodes in the output layer is generally based on the number of classification. If the network is used as classifier, the output layer nodes should be set the number of classes to be classified. Hidden layers can be used one, two or more hidden layers to construct the network. The more hidden layers of network, the mapping function is stronger, but the training time is expensive. If the number of hidden layer nodes is too little, the network will not have enough degrees of freedom to better fit the training data, resulting in the network is not strong or there are no any training results, more cannot recognize the samples, which are not trained previously, that is, fault tolerance is poor. If the number of hidden nodes are too much, the network training and learning time is too long, and the error is not necessarily optimal. In general, we can only rely on experience and testing to determine the hidden layer nodes.

\section{Energy Characterization Extraction Algorithm}

The original signal is denoted as $A_{s}$, according to the principle of wavelet decomposition, $A_{s}$ can be decomposed as:

$$
A_{s}=A_{1}+D_{1}=A_{2}+D_{1}+D_{2}=\cdots
$$

The decomposition of the reconstructed signal, which includes the time-domain of the original signal in different bands, so we can take advantage of this feature to extract the electric signal characteristics of the plant. Research under different frequency bands signal characteristics, and then extract the character frequency ranges, which can most express the fault features. The expression for the total energy of the signal is:

$$
E=E A_{j}+\sum E D_{j}
$$

The approximation signal of the j-th layer and the layers' detail signal energy can be selected as are preventatives to analyze the characteristics of the signal. The feature vector is constructed as following: $T=\left[E D_{1}, E D_{2}, \mathrm{~L}, E D_{j}\right]$. Feature extraction algorithm of the plant electric signal based on wavelet analysis is described as follows:

1) Conduct five-layer wavelet decomposition after de-noising a long period of time about 20s plant's electric signal, extracting the detail coefficients of wavelet band division, using $S_{i j}$ to represent wavelet coefficient of the $\mathrm{i}$-th layer $\mathrm{j}$-th node.

2)Calculate the variance and the norm of wavelet coefficients, according to the calculated results to class the experimental data.

3)Calculate the energy [14] of the wavelet coefficients, set the signal $S_{i j}$ corresponding to the energy is $E_{i j}$, then,

$$
E_{i j}=\int\left|S_{i j}(t)\right|^{2} d t=\sum_{k=1}^{n}\left|d_{i j}^{k}\right|^{2}
$$


Where, $d_{i j}^{k}$ represents sequence value of the $\mathrm{i}$-th layer $\mathrm{j}$-th node's wavelet coefficient.

4)Structure feature vector. Using five-layers of the wavelet decomposition energy value of detail wavelet coefficients as elements to structure feature vector $\mathrm{T}$, which can be described as follows:

$$
T=\left[E_{5,1}, E_{5,2}, E_{5,3}, E_{5,4}, E_{5,5}\right]
$$

Input the obtained feature vector to BP neural network to testing.

\section{Experimental Results and Analysis}

Four groups of plant electrical data referenced in this study are the aloe plant electrical signals from Xi'an University of Technology Laboratory collected. We'll represent the plant electrical signals collected under the darkness, smoke, broken branches and burning conditions using symbols: A, B, C, and D. Adopting db5 wavelet to conduct five layers wavelet decomposition for each plant electrical signal, extracting the wavelet coefficient of each node, calculating the norm and variance of wavelet coefficients as a kind of feature to analyze the signal. Because the sample data are more, so only 2 set of values of each characteristic group are listed in this paper, which are shown in Tables 2-3.

By contrast, it can be found that the characteristics of the data set A and B are in an order of magnitude, the characteristics of the data set $C$ and $D$ are in the other an order of magnitude, which usually higher than the previous. Because the four groups experimental data of aloe were collected in different environments, and the four environmental stimulate the aloe does not change by orders of magnitude. Obviously, the stimulation of aloe under the darkness and smoke conditions is weaker than it under the conditions of broken branches and burning. In order to make the classification accuracy of the plant electrical higher and the simulation experiments easier, we divided the experimental data into two categories: the signal under the conditions of darkness and smoke is a kind of class, the signal under the conditions of broken branches and burning is a kind of the other class. Through a lot of experimental results, it can easily distinguish these two categories.

Table 2. The Normal of Wavelet Coefficients

\begin{tabular}{cccccccc}
\hline Data Set & Number & $\mathrm{T} 1$ & $\mathrm{~T} 2$ & $\mathrm{~T} 3$ & $\mathrm{~T} 4$ & $\mathrm{~T} 5$ & $\mathrm{~T} 6$ \\
\hline \multirow{2}{*}{ A(Darkness) } & 1 & 267.4 & 3999 & 957.6 & 1202.2 & 1379.0 & 928.6 \\
& 2 & 325.1 & 574.0 & 1177.3 & 989.4 & 558.0 & 718.5 \\
B(Smoke) & 1 & 198.7 & 396.5 & 764.8 & 845.2 & 1144.2 & 1786.7 \\
C(broken & 2 & 271.3 & 596.5 & 836.0 & 1024.3 & 1282.2 & 636.9 \\
branches) & 2 & 10069 & 15992 & 16187 & 12882 & 7934 & 12275 \\
& 1 & 7215 & 11008 & 12708 & 15475 & 8101 & 15934 \\
D(Burning) & 2 & 7838 & 8664 & 9628 & 13027 & 10287 & 13521 \\
\hline
\end{tabular}

Table 3. The Variance of Wavelet Coefficients

\begin{tabular}{lcllcrrr}
\hline Data Set & Number & T1 & T2 & T3 & T4 & T5 & T6 \\
\hline \multirow{2}{*}{ A(Darkness) } & 1 & 18.955 & 28.342 & 67.880 & 85.223 & 97.631 & 63.308 \\
& 2 & 23.043 & 40.684 & 83.453 & 70.138 & 39.549 & 47.709 \\
B(Smoke) & 1 & 14.083 & 28.107 & 54.211 & 59.913 & 80.985 & 125.436 \\
& 2 & 19.231 & 42.284 & 59.259 & 72.551 & 90.658 & 45.037 \\
\hline
\end{tabular}




\begin{tabular}{cllllrrr}
\hline C(broken & 1 & 713.8 & 1133.7 & 1147.4 & 913.1 & 562.3 & 870.1 \\
branches) & 2 & 135.1 & 438.5 & 1147.1 & 596.7 & 379.1 & 449.2 \\
& 1 & 511.5 & 780.3 & 900.9 & 1096.8 & 574.3 & 1129.5 \\
D(Burning) & 2 & 555.62 & 614.15 & 682.48 & 923.43 & 729.16 & 955.04 \\
\hline
\end{tabular}

Furthermore, in order to identify the various electrical signal of aloe, calculate the energy of wavelet coefficients to construct a feature vector T, which is shown in Table 4.

BP neural network achieved successfully applied in many recognition and classification problems. In this paper, we adopt three-layer BP neural network to classify [15], which is widely used in the current. And input T to the BP neural network fortesting. Each category of data contains two the plant electrical, namely category vector includes two categories. Because the energy extracted feature vector dimension is 5 , so the BP neural network input layer nodes is 5. Based on empirical formulas and specific experimental results set the nodes of hidden layer is 12 and the nodes of output layer is 2 .

Table 4. Signal Energy Feature Extracted by Wavelet Algorithm

\begin{tabular}{|c|c|c|c|c|c|c|c|}
\hline Data Set & Number & $E_{5,1} E_{5,2} E$ & $E_{5,3} E_{5,4} E_{5,5}$ & & & & Category \\
\hline \multirow{2}{*}{ A(Darkness) } & 1 & 5.6683 & 1.6236 & 0.9377 & 10.3528 & 33.6125 & \multirow{4}{*}{ Class One } \\
\hline & 2 & 1.8990 & 0.5720 & 0.3149 & 3.0967 & 10.0626 & \\
\hline \multirow{2}{*}{$\mathrm{B}($ Smoke $)$} & 1 & 13.7896 & 3.7143 & 2.6730 & 36.9610 & 119.5506 & \\
\hline & 2 & 9.8822 & 2.5314 & 2.1087 & 26.7320 & 83.9371 & \\
\hline \multirow{2}{*}{$\begin{array}{l}\mathrm{C}(\text { Broken } \\
\text { branches })\end{array}$} & 1 & 0.6363 & 0.1392 & 0.0388 & 0.0761 & 0.5446 & \multirow{4}{*}{ Class Two } \\
\hline & 2 & 0.7220 & 0.1594 & 0.0651 & 0.1259 & 0.7621 & \\
\hline \multirow{2}{*}{ D(Burning) } & 1 & 0.8752 & 0.2377 & 0.1365 & 0.2852 & 1.5810 & \\
\hline & 2 & 1.5729 & 0.4655 & 0.1841 & 0.3001 & 2.4733 & \\
\hline
\end{tabular}

Neural network is an intelligent algorithm, each time the results are not necessarily able to achieve the set parameters. Setting the maximum number of training steps is 20000 , training target is 0.06 , and the program successfully reaches to set parameters denoting process efficiency. Training results are shown in Figure 4.

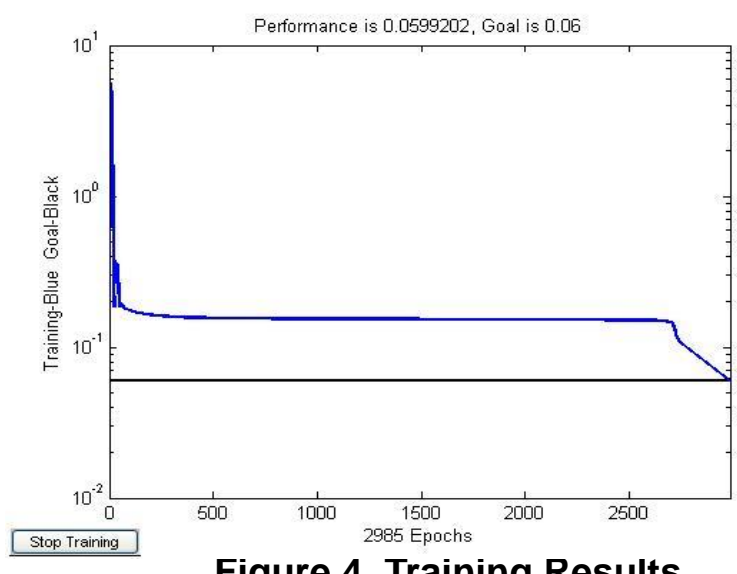

Figure 4. Training Results 
In the experiment, the plant electrical were conducted on 6-layer, 5-layer and 4-layer wavelet decomposition and obtaining each feature vector.

Input the feature vector, which was obtained by 6-layer wavelet decomposition, into $\mathrm{BP}$ neural network. Although the signal recognition rate is relatively higher, yet the program efficiency is relatively lower. Input the feature vector, which was obtained by 4-layer wavelet decomposition, into BP neural network. Although the program efficiency is relatively higher, yet the signal recognition rate is relatively lower. The results are shown in Table 5 .

Table 4. The Experimental Results of BP Neural Network

\begin{tabular}{ccc}
\hline The layers of Wavelet decomposition & Signal recognition rate & Program Efficiency \\
\hline 6 & $96 \%$ & $50 \%$ \\
5 & $91 \%$ & $83 \%$ \\
4 & $77 \%$ & $100 \%$ \\
\hline
\end{tabular}

Therefore, considering the experimental results, we selected the plant signal recognition method, which is based on the energy feature of 5-layer wavelet decomposition. The experimental results show that the plant electrical signal recognition rate of the proposed method reaches $91 \%$.

\section{Conclusion}

In this paper, the wavelet transform was carried out on the plant electrical signal decomposition. Choose the extracted norm and variance which include in different frequency bands of the signal, as the characteristics of preliminary identification of the plant electrical signal. Finally, a feature vector was input into BP neural network. The feature vector which was constructed by the energy values of the wavelet packet coefficient. The proposed method is only exploratory research in plant electrical aspects. In our further research work, other feature extraction methods and the improved BP neural network will be tried. At the same time, we will conduct different plant electrical simulation experiments. Therefore, the plant weak electrical signals study will play an increasingly important role in facility agriculture, the agricultural aspects of information technology. These methods have potential applications for monitoring plant responses to environmental change, for evaluating stress resistance, for physiological studies and for ecological/environmental monitoring.

\section{Acknowledgments}

This study was funded by the Science and Technology Research Key Project of Henan Education Department (14B416001) and Henan university of technology high-level talent funded project (2013BS063).

\section{References}

[1] Stankovib and Daviese, "Both action potentials and variation potentials induce proteinase inhibitor gene expression in tomato", FEBS Letters, vol. 390, no. 3, (1996).

[2] L. Chenghou, "In higher plants electrochemical wave messenger", ActaBiophysicaSinica, vol. 4, (1996).

[3] L. Gurovich and P. Hermosilla, "Electric signaling in fruit trees in response to water applications and light-darkness conditions", Plant Physiol, vol. 166, (2009).

[4] E. Davies, "New functions for electrical sigals in Plants", New Phytologist. vol. 161, (2004).

[5] S. M. Hanstein and H. H. Felle, "Nanoinfusion: an integrating tool to study elicitor perception and signal transduction in intact leaves", New Phytologist, vol. 161, (2004). 
[6] W. Lanzhou, L. Dongsheng and L. Jiao, "Advance on the testing research of weak electrical signals in plants", Journal of China Jiliang University, vol. 19, no. 1, (2008).

[7] W. Xu, X. Lifang, Y. Dan and H. Zhiyan, "Speech visualization based on wavelet transform", Application Research of Computers, vol. 26, no. 1, (2009).

[8] X. Yan, Z. Wang, L. Huang, C. Wang, R. Hou, Z. Xu and X. Qiao, "Research progress on electrical signals in higher plants", Progress in Natural Science, vol. 19, no. 5, (2009).

[9] C. Wei, W. Shaojie, W. Lingling, C. Chunhua and L. Hong, "Design of the measurement system for week electrical signal based of virtual instrument and wavelet de-noising", Chinese Agricultural Mechanization, vol. 231, no. 5, (2010).

[10] D. Jinli, D. Guiying and L. Haixial, "Study on Electrical Signals of Seedlings of Cucumissativus L. by a new Insecticide "ZJ0967"”, Journal of Zhejiang University of Science and Technology, vol. 18, no. 31, (2006).

[11] Z. Xiaohui, Y. Ningmei, X. Gang and M. Xiaoli, "Changes in the power spectrum of electrical signals in maize leafinduced by osmotic stress", Chinese Science Bullentin, vol. 57, no. 4, (2012).

[12] X. Zhang, N. Yu, X. Meng and G. Xi, "Power spectrum analysis of Maize based on wavelet de-noising", The 2nd international conference on information science and engineering, Hangzhou, China, (2010).

[13] L. Xiaobing, S. Xiaoli and X. Lianzhen, "Wavelet neural network in automatic target recognition based on wavelet moment", Journal of Southeast University(Natural Science Edition), vol. 1, (2006).

[14] Z. Yanqing and H. Yigang, "Fuzzy Neural Network Based Analog Circuit Fault Diagnosis Using Genetic Algorithms", Computer Science, vol. 37, no. 12, (2010).

[15] G. Zhexue and S. Zhiqiang, "Neural network theory and the realization of MATLAB", Publishing House of electronics industry, (2012). 
International Journal of Control and Automation Vol. 8, No. 7 (2015) 